\title{
Effect of hydrochlorothiazide in addition to telmisartan/amlodipine combination for treating hypertensive patients uncontrolled with telmisartan/amlodipine: a randomized, double-blind study
}

\author{
Jitsuo Higaki ${ }^{1}$, Issei Komuro ${ }^{2}$, Kosuke Shiki ${ }^{3}$, Ganghyuck Lee ${ }^{3}$, Atsushi Taniguchi ${ }^{3}$, Hiroshi Ikeda ${ }^{3}$, \\ Daisuke Kuroki ${ }^{3}$, Seiichiro Nishimura ${ }^{3}$ and Toshio Ogihara ${ }^{4}$
}

The efficacy and safety of telmisartan $80 \mathrm{mg} / \mathrm{amlodipine} 5 \mathrm{mg}$ plus hydrochlorothiazide $12.5 \mathrm{mg}$ (T80/A5/H12.5) was examined for its ability to treat hypertension in Japanese patients whose hypertension is uncontrolled with telmisartan 80 mg/amlodipine $5 \mathrm{mg}$ (T80/A5). Patients aged $\geqslant 20$ years who had essential hypertension despite taking two or three antihypertensive drugs entered a 6-week run-in period on T80/A5. Patients whose hypertension remained uncontrolled were randomly assigned to either the T80/A5/H12.5 group $(n=149)$ or the T80/A5 group $(n=160)$, once daily for 8 weeks. After 8 weeks, patients in the T80/A5/H12.5 group showed a significantly greater adjusted mean reduction in both seated diastolic blood pressure and seated systolic blood pressure than those in the T80/A5 group. Furthermore, more patients achieved a diastolic/systolic blood pressure of $<90 / 140 \mathrm{~mm} \mathrm{Hg}$ in the T80/A5/H12.5 group compared with the T80/A5 group. The most common adverse events were nasopharyngitis, elevated blood uric acid levels and hyperuricemia, and the latter two events were more frequent in the T80/A5/ $\mathrm{H} 12.5$ group than in the T80/A5 group. Overall, T80/A5/H12.5 administered for 8 weeks significantly reduced systolic and diastolic blood pressure and was well tolerated by patients with hypertension uncontrolled with T80/A5.

Hypertension Research (2017) 40, 251-258; doi:10.1038/hr.2016.124; published online 20 October 2016

Keywords: amlodipine; combination therapy; hydrochlorothiazide; telmisartan

\section{INTRODUCTION}

According to the Japanese Society of Hypertension Guidelines for the Management of Hypertension (JSH 2014), it is estimated that $\sim 43$ million people are affected by hypertension in Japan. ${ }^{1}$ It is concerning that the number of people with hypertension may continue to increase with aging of the population. In a survey of 4000 hypertensive subjects, the proportion with controlled blood pressure was 34.9\% in 2006, and this improved to $50 \%$ in 2009 ; however, blood pressure control remains inadequate. ${ }^{2}$ Another survey conducted in 3000 subjects in 1994, 2001 and 2010 showed that the mean number of prescribed agents per patient increased gradually from 1.64 in 1994 to 1.79 in 2001 and to 2.04 in $2010 .{ }^{3}$ For many patients, a combination of two or more treatments, or an increased dose of combination therapy, is required to achieve any antihypertensive effect. ${ }^{1}$
Angiotensin II receptor blockers (ARBs), angiotensin-converting enzyme inhibitors, calcium channel blockers (CCBs) and diuretics are first-line drugs for the management of hypertension. ${ }^{1}$ CCBs and ARBs lower blood pressure by vasodilating effects. Diuretics increase the excretion of excess water and sodium, thereby decreasing peripheral vascular resistance.

Among these first-line drugs, $\mathrm{ARB} / \mathrm{CCB}$ combination therapy is widely used for hypertensive patients as a second-line treatment. In a phase III study, a combined antihypertension treatment containing $80 \mathrm{mg}$ of the $\mathrm{ARB}$ telmisartan and $5 \mathrm{mg}$ of the $\mathrm{CCB}$ amlodipine (Micamlo combination tablets BP; Nippon Boehringer Ingelheim) reduced diastolic blood pressure (DBP) to $\leqslant 90 \mathrm{~mm} \mathrm{Hg}$ in $70.1 \%$ of patients compared with $41.5 \%$ of patients taking telmisartan monotherapy (data on file, Nippon Boehringer Ingelheim, Tokyo, Japan).

${ }^{1}$ Department of Cardiology, Pulmonology, Hypertension and Nephrology, Ehime University Graduate School of Medicine, Ehime, Japan; ${ }^{2}$ Department of Cardiovascular Medicine, Graduate School of Medicine, The University of Tokyo, Tokyo, Japan; ${ }^{3}$ Medical Division, Nippon Boehringer Ingelheim, Tokyo, Japan and ${ }^{4}$ Morinomiya University of Medical Sciences, Osaka, Japan

Correspondence: Dr J Higaki, Department of Cardiology, Pulmonology, Hypertension and Nephrology, Ehime University Graduate School of Medicine, Shitsukawa, Toon, Ehime 791-0295, Japan.

E-mail: jhigaki@m.ehime-u.ac.jp

Received 20 April 2016; revised 23 July 2016; accepted 18 August 2016; published online 20 October 2016 
For patients with hypertension refractory to $\mathrm{ARB} / \mathrm{CCB}$, the use of an additional antihypertensive drug with a different mode of action, such as a low dose of a diuretic, is recommended. ${ }^{1}$ However, diuretics are infrequently used to treat Japanese hypertensive patients. We designed this study to determine the effects of adding the diuretic hydrochlorothiazide to an ARB/CCB combination therapy.

The aim of this prospective, randomized, double-blind, active-control, parallel-group comparison study was to examine the efficacy and safety of telmisartan $(80 \mathrm{mg})$, amlodipine $(5 \mathrm{mg})$ and hydrochlorothiazide $(12.5 \mathrm{mg})$ (T80/A5/H12.5) for the treatment of hypertensive patients whose hypertension is uncontrolled with telmisartan (80 mg) and amlodipine (5 mg) (T80/A5).

\section{MATERIALS AND METHODS}

\section{Patients}

Japanese patients aged $\geqslant 20$ years were enrolled in this study if they had essential hypertension, were already taking two or three antihypertensive drugs (at the time of the visit for signing the informed consent form), had a mean (of three measurements in one visit) seated DBP $\geqslant 90$ and $\leqslant 114 \mathrm{~mm} \mathrm{Hg}$ at week -6 and week 0 (see Figure 1 for details of visits), had a mean seated systolic blood pressure (SBP) $\leqslant 200 \mathrm{~mm} \mathrm{Hg}$ at week -6 and week 0 , were an outpatient, were able to stop all current antihypertensive drugs (other than study medication) from week -6 through to the end of the study without risk and if they provided informed consent. Patients were excluded if they had any of the following: known or suspected secondary hypertension, cardiac disorders, a history of stroke or transient ischemic attack within the past 6 months, hepatic or renal dysfunctions, biliary atresia or cholestasis, hyperkalemia, anuria or blood dialysis, hyponatremia, malignant tumor or a disease requiring immunosuppressants. Patients were also excluded if they were $<80 \%$ or $>120 \%$ compliant during the run-in period. The study was conducted in accordance with the principles of the Declaration of Helsinki. Ethical approval was acquired from all institutional review boards and all patients provided written informed consent that they were free to withdraw at any time during the study.

Prescribed treatments for concomitant diseases, except for those known to affect blood pressure, were not discontinued during the study. Patient compliance was measured at each visit by counting the number of tablets returned to the investigation and calculating the percentage of medication taken in relation to the amount that should have been taken.

\section{Study design}

This study was designed as an 8-week, multicenter (30 sites), randomized, double-blind, active-control, parallel-group comparative phase III clinical study conducted between 8 November 2013 and 12 July 2014 to verify the superiority of T80/A5/H12.5 over T80/A5 for the reduction in blood pressure. Patients entered a 6-week open-label run-in period that involved the oral administration of a once-daily T80/A5 fixed-dose combination tablet. At the end of the run-in

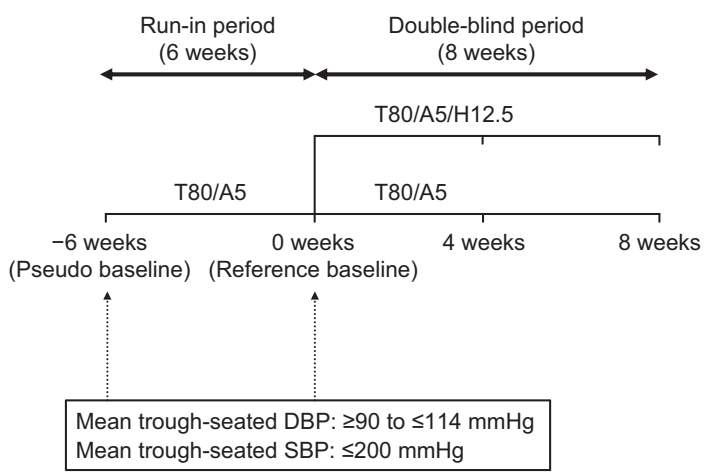

Figure 1 Study design. DBP, diastolic blood pressure; SBP, systolic blood pressure; T80/A5, telmisartan $80 \mathrm{mg} /$ amlodipine $5 \mathrm{mg}$; T80/A5/H12.5, telmisartan $80 \mathrm{mg} /$ amlodipine $5 \mathrm{mg}+$ hydrochlorothiazide $12.5 \mathrm{mg}$. period (week 0), patients whose trough-seated DBP remained $\geqslant 90 \mathrm{~mm} \mathrm{Hg}$ after treatment with T80/A5 were randomly assigned to one of two groups: once-daily, orally administered T80/A5 fixed-dose combination tablet+H12.5 (T80/A5/H12.5) or T80/A5 fixed-dose combination tablet+placebo (T80/A5). The treatment period lasted 8 weeks (Figure 1). Randomization and appropriate supply of study medication to patients was performed using Interactive Response Technology.

\section{Study endpoints}

Efficacy. The primary endpoint of this study was the reduction from the reference baseline in mean seated DBP at trough after 8 weeks of the double-blind period. The key secondary endpoint was the reduction from the reference baseline in mean seated SBP at trough after 8 weeks of the double-blind period. The other secondary endpoint was the proportion of patients with seated DBP $<90 \mathrm{~mm} \mathrm{Hg}$ and seated SBP $<140 \mathrm{~mm} \mathrm{Hg}$ at trough after 8 weeks of the double-blind period. Other endpoints included the seated DBP control rate (percentage of patients with DBP $<90 \mathrm{~mm} \mathrm{Hg}$ ) and SBP control rate (percentage of patients with SBP $<140 \mathrm{~mm} \mathrm{Hg}$ ) at trough after 8 weeks of the double-blind period and seated DBP response rate (percentage of patients with DBP $<90 \mathrm{~mm} \mathrm{Hg}$ or a reduction in DBP of $\geqslant 10 \mathrm{~mm} \mathrm{Hg}$ ) and SBP response rate (percentage of patients with SBP $<140 \mathrm{~mm} \mathrm{Hg}$ or a reduction in SBP of $\geqslant 20 \mathrm{~mm} \mathrm{Hg}$ ) at trough after 8 weeks of the double-blind period from the reference baseline.

Blood pressure and heart rate measurements. All blood pressure measurements were taken with a standard mercury sphygmomanometer, recorded on the same arm by the same operator where possible. Blood pressure and pulse rate at trough were measured $\sim 24 \mathrm{~h}( \pm 3 \mathrm{~h})$ after the last dose of the study drugs on the previous day. Seated blood pressure was calculated as the mean of three measurements.

Seated blood pressure and pulse rate were measured at weeks $-6,0,4$ and 8 . Blood pressure and pulse rate were measured in seated, supine and standing positions at weeks $-6,0$ and 8 . DBP was taken at the disappearance of repetitive sound (Korotkoff phase 5). In those in whom the sound continued until the zero point, the blood pressure at the distinct muffling of the repetitive sounds (Korotkoff phase 4) was to be recorded as the DBP.

Safety. To ensure the safety of the study participants, the parameters were analyzed during the study, while maintaining blind, to identify potential safety concerns, including the incidence of adverse events, changes in blood pressure and pulse rate following position change, seated pulse rate and general laboratory tests (blood biochemistry, hematology and urinalysis).

\section{Statistical analyses}

The sample size of 300 (150 per group; allocation ratio 1:1) was determined to achieve superiority of the T80/A5/H12.5 group to the T80/A5 group with two-sided significance level of $5 \%$ and power of $>80 \%$ provided that the seated DBP reduction would differ between treatment groups by $2.8 \mathrm{~mm} \mathrm{Hg}$ with s.d. of $8.5 \mathrm{~mm} \mathrm{Hg}$.

In this study, three analysis sets were defined for analyzing data, including the treated set, the full analysis set (FAS) and the per-protocol set (PPS). The treated set was defined as all patients (1) randomly assigned to one of two treatment groups and (2) taking at least one dose of T80/A5/H12.5 or T80/A5. The FAS was defined as all patients (1) included in the treated set and (2) taking measurements of seated DBP at reference baseline and at one or more time points during the double-blind period. The PPS was defined as a collection of patients (1) included in the FAS and (2) observing no important protocol violation that might affect the efficacy evaluation during the double-blind period.

For the primary and key secondary endpoints, an analysis of covariance (ANCOVA) was performed on the FAS to analyze the blood pressure reduction after 8 weeks of treatment in the T80/A5/H12.5 and T80/A5 groups. The model includes treatment and center as fixed effects, and reference baseline as a covariate. The last observation carried forward (LOCF) approach was used to impute missing data. The treatment effect was tested and estimated by the adjusted mean and its 95\% confidence interval (CI) obtained from the model. The sensitivity analyses were performed using an ANCOVA on the PPS, and 
using mixed-effects model repeated measures (MMRM) on the FAS without the LOCF approach. The other secondary endpoint and other endpoints, including the proportion of patients with trough-seated DBP/SBP $\leqslant 90 / 140 \mathrm{~mm} \mathrm{Hg}$, DBP/SBP control rates and DBP/SBP response rates after 8 weeks of the double-blind period, were analyzed by treatment group and compared between groups using logistic regression analysis with noncompleters considered failure imputation. (Clinical trial registration: NCT01975246.)

\section{RESULTS}

\section{Patients}

We enrolled 442 male and female Japanese patients in this study. Of these, 309 patients had uncontrolled blood pressure despite treatment with T80/A5 during the run-in period (Figure 2). The study population consisted predominantly of males (83.2\%) and people aged $<65$ years $(85.4 \%)$, with the mean (s.d.) age of all patients being 54.7 (9.4) years (Table 1). The majority of patients had hypertension severity grade I or II ( $72.8 \%$ and $24.6 \%$, respectively) according to JSH 2014. ${ }^{1}$ The mean (s.d.) body mass index of the study population was 26.92 (4.34) $\mathrm{kg} \mathrm{m}^{-2}$ and the mean (s.d.) waist circumference was $92.90(10.76) \mathrm{cm}$. In total, $73.8 \%$ of the treated patients had abdominal obesity. Overall, of the 309 patients in the treated set, 93.5\% had at least one concomitant diagnosis; the most frequent was metabolic and nutrition disorders (72.2\%), followed by hepatobiliary disorders $(27.8 \%)$.

In accordance with the inclusion criteria, patients were previously treated with two $(82.2 \%)$ or three $(17.8 \%)$ antihypertensive drugs. In $74.4 \%$ of patients, this consisted of an $\mathrm{ARB}$ and a CCB, and for $10.7 \%$ it consisted of an ARB, a CCB and a diuretic. The mean (s.d.) seated DBP at reference baseline was 96.5 (5.6) $\mathrm{mm} \mathrm{Hg}$ and $95.7(5.1 \mathrm{~mm} \mathrm{Hg})$ in the T80/A5/H12.5 and the T80/A5 groups,

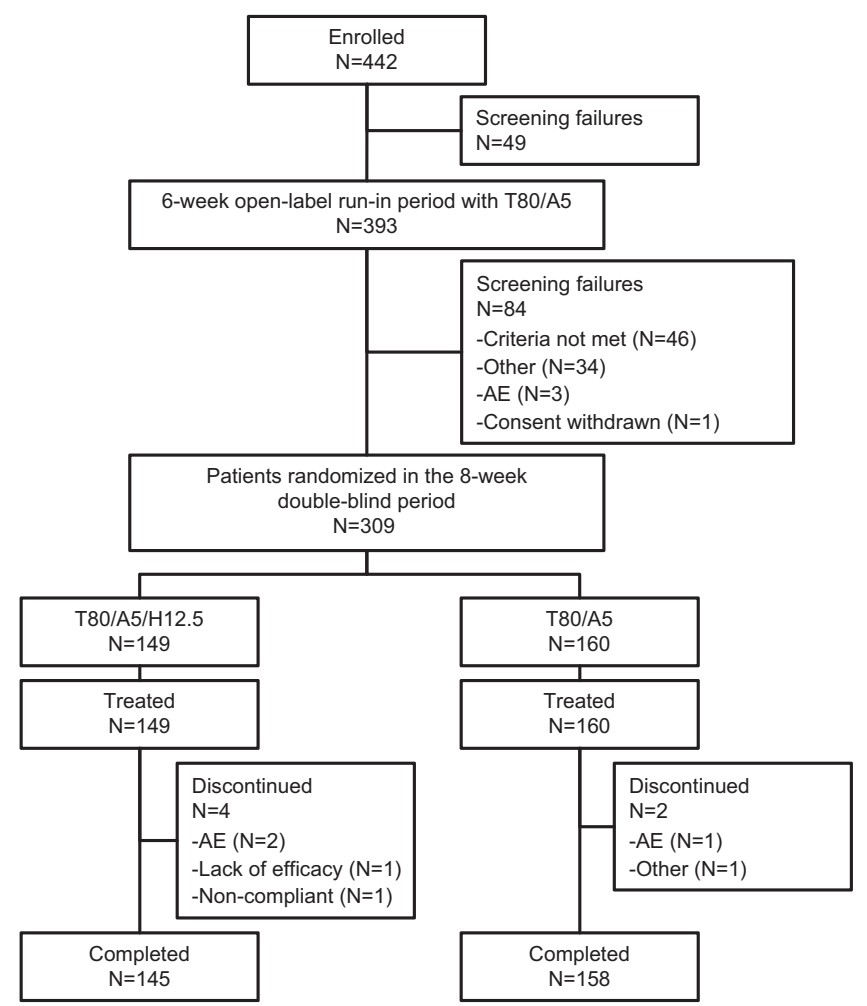

Figure 2 Patient disposition. AE, adverse event; T80/A5, telmisartan $80 \mathrm{mg}$ +amlodipine $5 \mathrm{mg}$; T80/A5/H12.5, telmisartan $80 \mathrm{mg} /$ amlodipine $5 \mathrm{mg}$ +hydrochlorothiazide $12.5 \mathrm{mg}$. respectively. The mean seated SBP at reference baseline was 142.3 (12.9) and 142.3 (12.1) $\mathrm{mm} \mathrm{Hg}$ in the T80/A5/H12.5 and the T80/A5 groups, respectively. In general, the demographic and reference baseline characteristics of both groups were similar (Table 1).

The disposition of the study patients is shown in Figure 2. Of the 442 enrolled patients, 393 entered the run-in period and 49 were excluded, largely because of violations of the inclusion/exclusion criteria. Following the run-in period, 309 patients were randomly assigned to T80/A5/H12.5 $(N=149)$ and T80/A5 $(N=160)$ groups and 84 were excluded, mainly because of not meeting the blood pressure inclusion/exclusion criteria. Of the 309 patients who entered the treatment period, $303(98.1 \%)$ patients completed the 8 -week treatment period, and the proportion of patients who completed the study was similar between groups (145 [97.3\%] and 158 [98.8\%] for the T80/A5/H12.5 and T80/A5 groups, respectively). The most frequent cause for discontinuation was adverse events that occurred in $2(1.3 \%)$ and $1(0.6 \%)$ patients in the T80/A5/H12.5 and T80/A5 groups, respectively (Figure 2).

\section{DBP change}

After 8 weeks of treatment, the adjusted mean (s.e.) reduction in trough-seated DBP was $8.4(0.6) \mathrm{mm} \mathrm{Hg}$ in the T80/A5/H12.5 group compared with $4.5(0.5) \mathrm{mm} \mathrm{Hg}$ in the T80/A5 group in the FAS (ANCOVA with LOCF). The T80/A5/H12.5 group showed a statistically significantly greater adjusted mean reduction in seated DBP compared with the T80/A5 treatment group, with a difference of $-3.9 \mathrm{~mm} \mathrm{Hg}$ (95\% CI: -5.3 to $-2.4 ; P<0.0001)$. Figure 3 a shows the results of a sensitivity analysis performed using a MMRM on the FAS that provided results that were consistent with those of the primary analysis. An ANCOVA on the PPS also provided results consistent with those of the primary analysis. In addition, the adjusted mean (s.e.) change from reference baseline in trough-seated DBP at week 4 based on the MMRM analysis was $-6.6(0.5) \mathrm{mm} \mathrm{Hg}$ in the T80/A5/H12.5 group and $-3.2(0.5) \mathrm{mm} \mathrm{Hg}$ in the T80/A5 group. Compared with T80/A5, T80/A5/H12.5 had a greater reduction in trough-seated DBP, with a treatment difference of $-3.5 \mathrm{~mm} \mathrm{Hg}(95 \% \mathrm{CI}:-4.8$ to -2.1 ; $P<0.0001)$. These data indicate that treatment with the combination T80/A5/H12.5 achieved a superior reduction in seated DBP compared with treatment with T80/A5.

\section{SBP change}

Following 8 weeks of treatment, the adjusted mean (s.e.) reduction in trough-seated SBP was $12.3(0.8) \mathrm{mm} \mathrm{Hg}$ in the T80/A5/H12.5 group compared with $6.9(0.8) \mathrm{mm} \mathrm{Hg}$ in the T80/A5 group in the FAS (ANCOVA with LOCF). The T80/A5/H12.5 group showed a statistically significantly greater adjusted mean reduction in SBP compared with the T80/A5 group, with a difference of $-5.3 \mathrm{~mm} \mathrm{Hg}$ (95\% CI: -7.6 to $-3.1 ; P<0.0001$ ). The sensitivity analysis, including a MMRM analysis on the FAS (Figure $3 \mathrm{~b}$ ), provided results that were consistent with those of the primary analysis. Furthermore, the adjusted mean (s.e.) change from reference baseline in trough-seated SBP at week 4 based on the MMRM analysis was $-9.7(0.8) \mathrm{mm} \mathrm{Hg}$ in the T80/A5/H12.5 group and $-5.2(0.8) \mathrm{mm} \mathrm{Hg}$ in the T80/A5 group. Compared with the T80/A5 group, the T80/A5/H12.5 group had a greater reduction in trough-seated SBP, with a treatment difference of $-4.5 \mathrm{~mm} \mathrm{Hg}$ (95\% CI: -6.6 to $-2.3 ; P<0.0001$; MMRM analysis; Figure $3 \mathrm{~b})$. These data indicate that treatment with the combination T80/A5/H12.5 achieved a superior reduction in SBP compared with treatment with T80/A5. 
Table 1 Demographic and reference baseline characteristics of the treated set

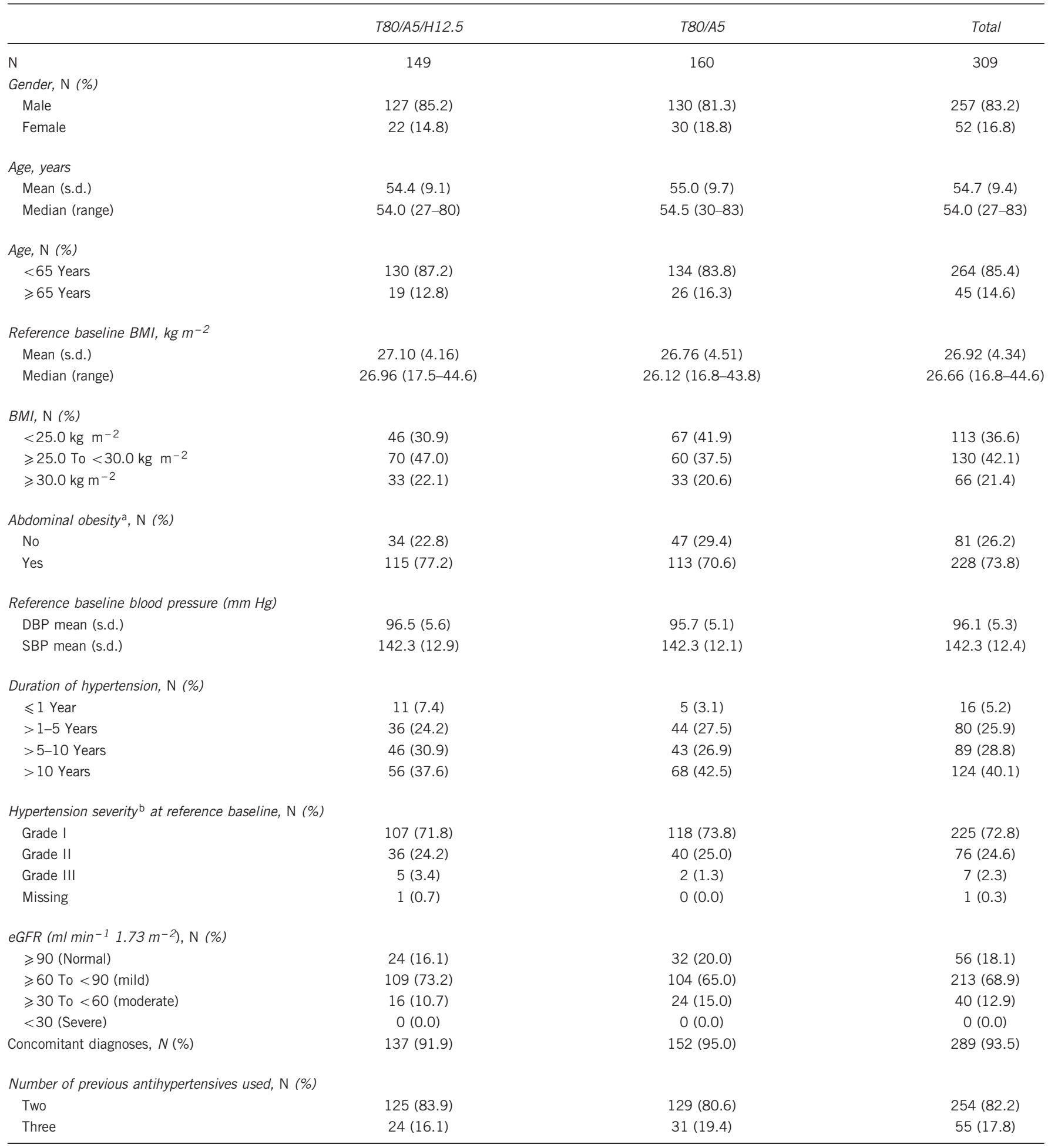

Abbreviations: BMI, body mass index; DBP, diastolic blood pressure; eGFR, estimated glomerular filtration rate; SBP, systolic blood pressure; T80/A5, telmisartan 80 mg/amlodipine 5 mg; T80/A5/H12.5, telmisartan $80 \mathrm{mg} /$ amlodipine $5 \mathrm{mg}+$ hydrochlorothiazide $12.5 \mathrm{mg}$.

a Abdominal obesity: Yes $=$ reference baseline waist circumference $>85 \mathrm{~cm}$ (male) and $>90 \mathrm{~cm}$ (female).

bHypertension severity: grade I= seated SBP 140 to $<160 \mathrm{~mm} \mathrm{Hg}$ or seated DBP 90 to $<100 \mathrm{~mm} \mathrm{Hg}$; Grade II=seated SBP 160 to $<180 \mathrm{~mm} \mathrm{Hg}$ or seated DBP 100 to $<110 \mathrm{~mm} \mathrm{Hg}$; Grade III = seated SBP $\geqslant 180 \mathrm{~mm} \mathrm{Hg}$ or seated DBP $\geqslant 110 \mathrm{~mm} \mathrm{Hg}$.

\section{Other outcomes}

Following 8 weeks of treatment, the proportion of patients who achieved a trough-seated DBP/SBP of $<90 / 140 \mathrm{~mm} \mathrm{Hg}$ was $51.7 \%$ in the T80/A5/H12.5 group and 36.9\% in the T80/A5 group (Table 2).
Based on logistic regression analysis, patients in the T80/A5/H12.5 group were estimated to be 2.1 times more likely to achieve a DBP/SBP of $<90 / 140 \mathrm{~mm} \mathrm{Hg}$ than were patients in the T80/A5 group (95\% CI: 1.2 to 3.4 ). In addition, more patients achieved 
a

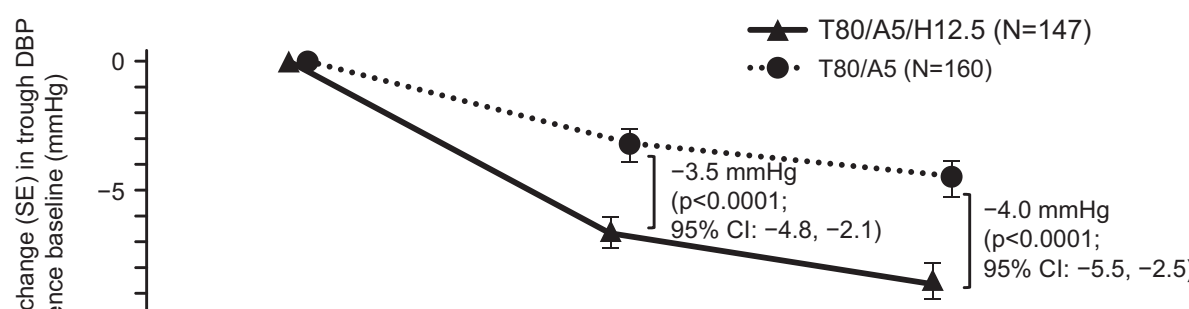

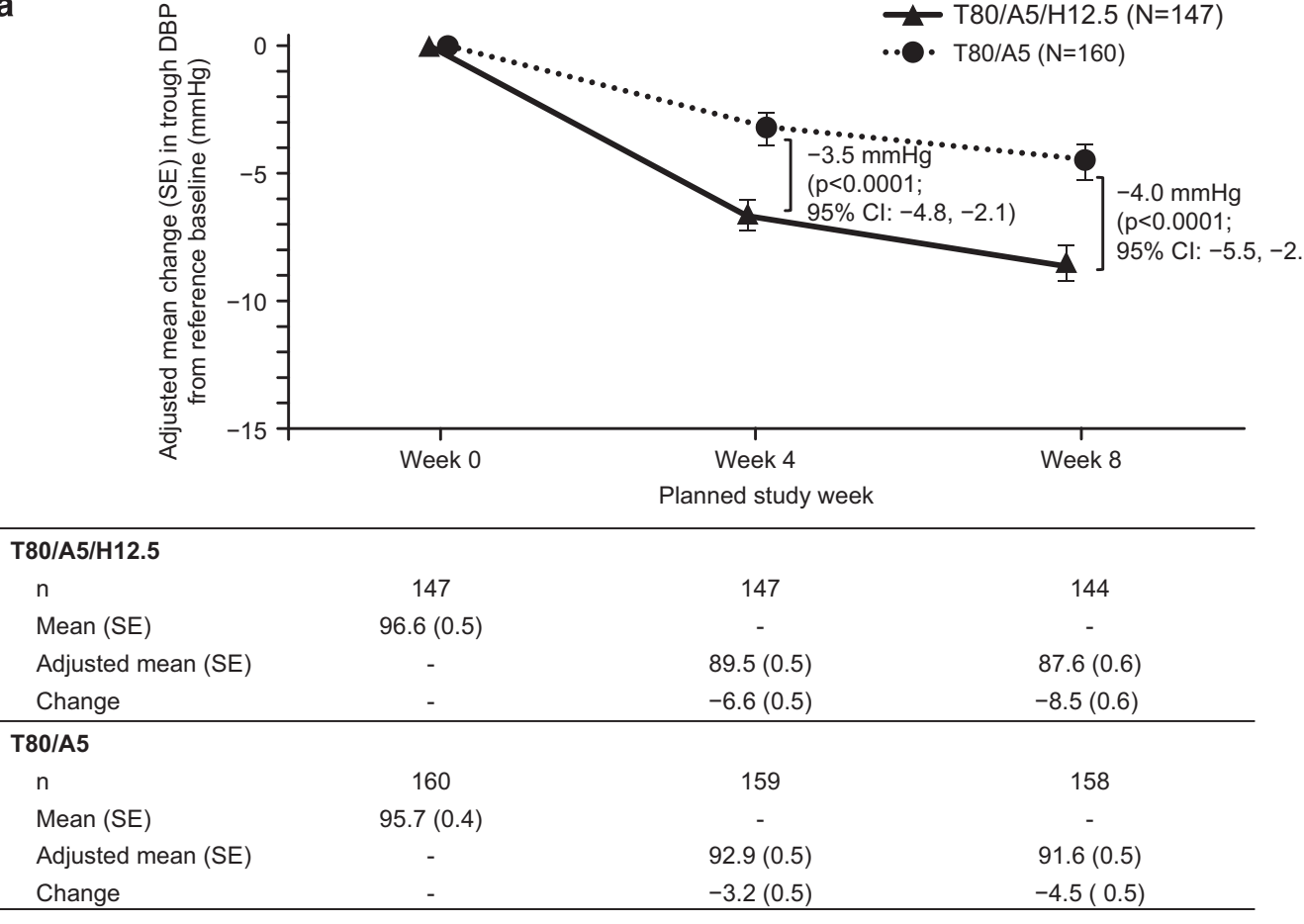

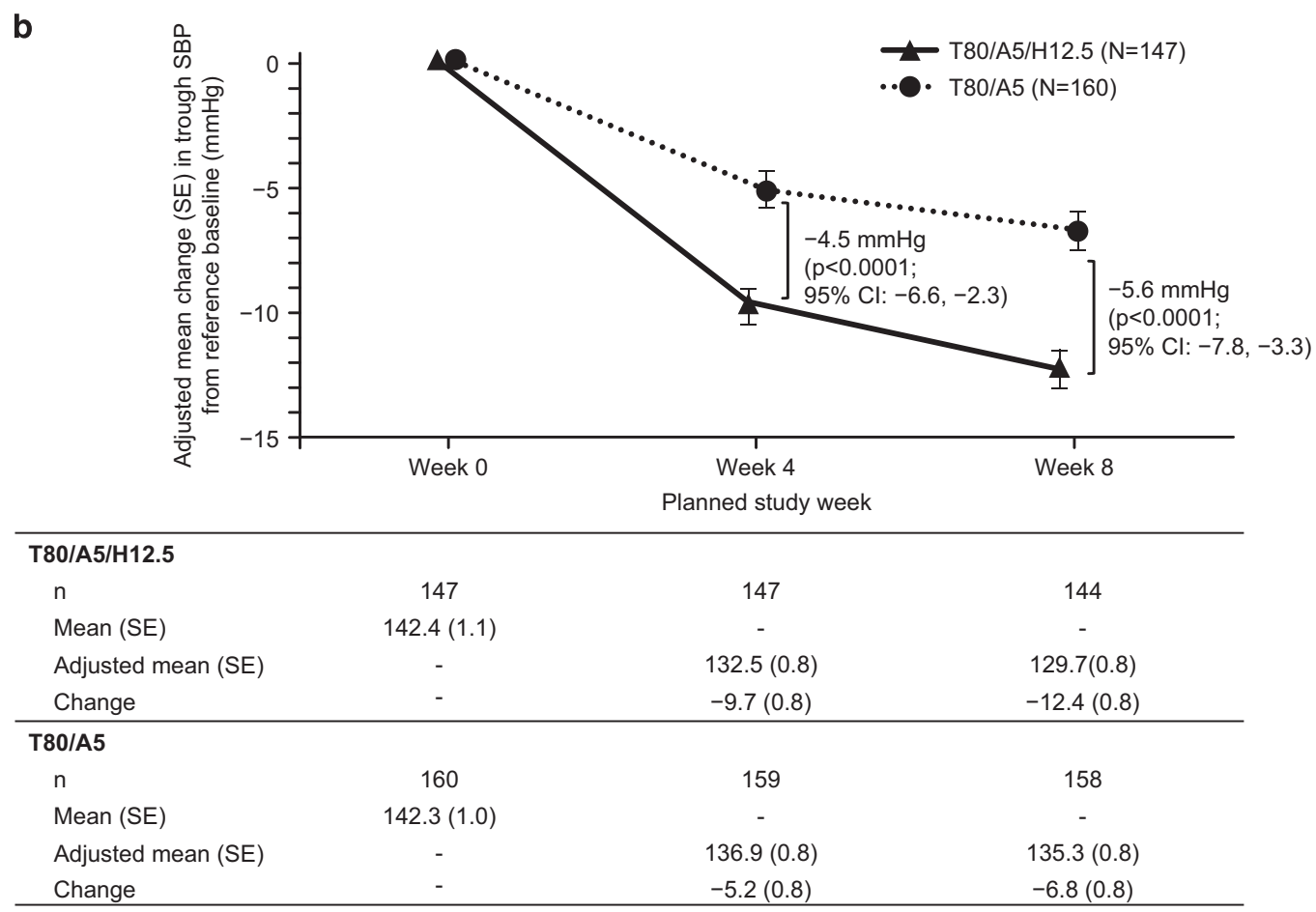

Figure 3 Change in trough-seated DBP and SBP from reference baseline using a mixed-effects model for repeated measures on the full analysis set with no imputation. (a) Adjusted mean change in DBP compared with reference baseline at 4 and 8 weeks for both treatment groups in the FAS. (b) Adjusted mean change in SBP compared with reference baseline at 4 and 8 weeks of treatment for both treatment groups in the FAS. DBP, diastolic blood pressure; $\mathrm{SBP}$, systolic blood pressure; T80/A5, telmisartan $80 \mathrm{mg}+$ amlodipine $5 \mathrm{mg}$; T80/A5/H12.5, telmisartan 80 mg+amlodipine 5 mg+hydrochlorothiazide $12.5 \mathrm{mg}$.

trough-seated DBP control (DBP $<90 \mathrm{~mm} \mathrm{Hg}$ ) in the T80/A5/H12.5 group than in the T80/A5 group (Table 2). The probability of achieving DBP control at week 8 was estimated to be 1.9 times higher in the T80/A5/H12.5 group than in the T80/A5 group (95\% CI: 1.2 to 3.2) on the basis of a logistic regression analysis.
Of the patients within the FAS who had SBP $\geqslant 140 \mathrm{~mm} \mathrm{Hg}$ at reference baseline, a greater portion achieved trough-seated SBP control (SBP $<140 \mathrm{~mm} \mathrm{Hg}$ ) by week 8 in the T80/A5/H12.5 group compared with patients in the T80/A5 group (Table 2). The probability of achieving SBP control at week 8 was estimated to be 2.5 times higher 
Table 2 DBP and SBP control rates and response rates in the full analysis set

\begin{tabular}{|c|c|c|c|c|c|c|c|c|c|}
\hline \multirow[b]{2}{*}{ Endpoint } & \multirow[b]{2}{*}{ Week } & \multicolumn{3}{|c|}{ T80/A5/H12.5 } & \multicolumn{3}{|c|}{ T80/A5 } & \multirow[b]{2}{*}{$O R^{a}$} & \multirow[b]{2}{*}{$95 \% \mathrm{Cl}$} \\
\hline & & $N$ & $\mathrm{n}$ & (\%) & $\mathrm{N}$ & $n$ & (\%) & & \\
\hline \multirow[t]{2}{*}{ DBP control } & 4 & 147 & 70 & 47.6 & 160 & 47 & 29.4 & 2.4 & (1.5-4. \\
\hline & 8 & & 83 & 56.5 & & 68 & 42.5 & 1.9 & $(1.2-3.2)$ \\
\hline \multirow[t]{2}{*}{ SBP control } & 4 & 76 & 37 & 48.7 & 89 & 27 & 30.3 & 2.3 & $(1.2-4.8)$ \\
\hline & 8 & & 48 & 63.2 & & 40 & 44.9 & 2.5 & $(1.2-5.3)$ \\
\hline DBP $<90 \mathrm{~mm} \mathrm{Hg}$ & 4 & 147 & 80 & 54.4 & 160 & 49 & 30.6 & 3.0 & $(1.8-5$. \\
\hline $\begin{array}{l}\text { or decrease by } \\
10 \mathrm{~mm} \mathrm{Hg}\end{array}$ & 8 & & 98 & 66.7 & & 72 & 45.0 & 2.8 & $(1.7-4.8)$ \\
\hline SBP $<140 \mathrm{~mm} \mathrm{Hg}$ & 4 & 76 & 41 & 53.9 & 89 & 30 & 33.7 & 2.4 & $(1.2-4.8)$ \\
\hline $\begin{array}{l}\text { or decrease by } \\
20 \mathrm{~mm} \mathrm{Hg}\end{array}$ & 8 & & 54 & 71.1 & & 40 & 44.9 & 4.1 & $(1.9-9.1)$ \\
\hline $\mathrm{DBP} / \mathrm{SBP}$ & 4 & 147 & 66 & 44.9 & 160 & 39 & 24.4 & 2.9 & $(1.7-5.1)$ \\
\hline$<90 / 140 \mathrm{~mm} \mathrm{Hg}$ & 8 & & 76 & 51.7 & & 59 & 36.9 & 2.1 & $(1.2-3$. \\
\hline
\end{tabular}

Abbreviations: $\mathrm{Cl}$, confidence interval; DBP, diastolic blood pressure; OR, odds ratio;

$\mathrm{SBP}$, systolic blood pressure; T80/A5, telmisartan 80 mg/amlodipine 5 mg; T80/A5/H12.5, telmisartan $80 \mathrm{mg} /$ amlodipine $5 \mathrm{mg}+$ hydrochlorothiazide $12.5 \mathrm{mg}$.

Only patients in the full analysis set with a trough-seated DSB of $\geqslant 90 \mathrm{~mm} \mathrm{Hg}$ or a SBP of

$\geqslant 140 \mathrm{~mm} \mathrm{Hg}$ at reference baseline were included in the analysis.

aFor T80/A5/H12.5 vs. T80/A5.

in the T80/A5/H12.5 group than in the T80/A5 group (95\% CI: 1.2 to 5.3) on the basis of a logistic regression analysis. Furthermore, the proportion of patients who had an adequate response in trough-seated DBP (DBP $<90 \mathrm{~mm} \mathrm{Hg}$ or a reduction in DBP of $\geqslant 10 \mathrm{~mm} \mathrm{Hg}$ ) at week 8 was larger in the T80/A5/H12.5 group than in the T80/A5 group. Of the patients within the FAS who had SBP $\geqslant 140 \mathrm{~mm} \mathrm{Hg}$ at reference baseline, more had an adequate response in trough-seated SBP (SBP $<140 \mathrm{~mm} \mathrm{Hg}$ or a reduction in SBP of $\geqslant 20 \mathrm{~mm} \mathrm{Hg}$ ) at week 8 in the T80/A5/H12.5 group than in the T80/A5 group.

\section{Subgroup analysis}

Within the treatment groups, patients were divided into subgroups based on patient characteristics, including age $(<65, \geqslant 65$ years), sex, body mass index $\left(<25,25-<30\right.$ and $\left.\geqslant 30 \mathrm{~kg} \mathrm{~m}^{-2}\right)$, hypertension severity, hypertension duration $(\leqslant 1,>1-5,>5-10$ and $>10$ years) and the number of previous antihypertension drugs used (two or three). The reductions in both DBP and SBP in each subgroup tended to be greater in patients treated with T80/A5/H12.5 than in patients treated with T80/A5 (Supplementary Tables 1 and 2).

\section{Safety}

Adverse events and laboratory findings. The number of patients who experienced adverse events during the double-blind 8-week period was higher in the T80/A5/H12.5 group (41.6\%) than in the T80/A5 group (28.1\%) (Table 3). The most common adverse events were increased blood uric acid levels, nasopharyngitis and hyperuricemia. Higher incidences in the T80/A5/H12.5 group compared with the T80/A5 group were seen for increased blood uric acid levels and hyperuricemia.

The frequencies of drug-related adverse events are shown in Table 3. The incidences of drug-related adverse events were higher in the T80/A5/H12.5 group (23.5\%) compared with the T80/A5 group $(3.8 \%)$. This difference in the number of drug-related adverse events
Table 3 Summary of AEs and frequency of drug-related AEs experienced by $\geqslant 1 \%$ of the patients in any one of the treatment groups in the treated set

\begin{tabular}{|c|c|c|c|c|}
\hline \multirow[b]{2}{*}{ Variable } & \multicolumn{2}{|c|}{ T80/A5/H12.5 } & \multicolumn{2}{|c|}{ T80/A5 } \\
\hline & $\mathrm{N}$ & $\%$ & $\mathrm{~N}$ & $\%$ \\
\hline Number of patients & 149 & 100 & 160 & 100 \\
\hline Patients with an $\mathrm{AE}$ & 62 & 41.6 & 45 & 28.1 \\
\hline Patients with investigator-defined drug-related AEs & 35 & 23.5 & 6 & 3.8 \\
\hline Patients with other significant AEs & 2 & 1.3 & 1 & 0.6 \\
\hline Patients with AEs leading to discontinuation & 2 & 1.3 & 1 & 0.6 \\
\hline \multicolumn{5}{|l|}{ System organ class and preferred term } \\
\hline Metabolic and nutrition disorders & 10 & 6.7 & 1 & 0.6 \\
\hline Hyperuricemia & 8 & 5.4 & 1 & 0.6 \\
\hline Dyslipidemia & 2 & 1.3 & 0 & 0.0 \\
\hline Investigations (laboratory data abnormalities) & 21 & 14.1 & 4 & 2.5 \\
\hline Blood uric acid increased & 20 & 13.4 & 3 & 1.9 \\
\hline Blood creatinine increased & 2 & 1.3 & 0 & 0.0 \\
\hline Blood urea increased & 2 & 1.3 & 0 & 0.0 \\
\hline
\end{tabular}

Abbreviations: AE, adverse event; T80/A5, telmisartan 80 mg+amlodipine 5 mg; T80/A5/H12.5, telmisartan 80 mg+amlodipine 5 mg+hydrochlorothiazide $12.5 \mathrm{mg}$.

was largely because of the higher incidence of increased uric acid levels in the T80/A5/H12.5 group compared with the T80/A5 group (13.4\% vs. $1.9 \%)$.

Indeed, regarding laboratory parameters (Supplementary Table 3), noteworthy differences between groups were observed for the mean change from reference baseline in uric acid $\left(1.0 \mathrm{mg} \mathrm{dl}^{-1}\right.$ in the T80/A5/H12.5 group $v s .0 .2 \mathrm{mg} \mathrm{dl}^{-1}$ in the T80/A5 group). In patients who had no abnormalities at reference baseline, possible clinically relevant abnormalities included the development of high triglycerides and high total cholesterol that were detected in $11.2 \%$ and $4.3 \%$ of patients in the T80/A5/H12.5 group, respectively, and $7.3 \%$ and $5.9 \%$ of the patients in the T80/A5 group, respectively.

The adverse events experienced by patients were mild or moderate in severity and no severe adverse events or deaths were reported in either treatment group. Two patients in the T80/A5/H12.5 group discontinued the study because of adverse events: atrial fibrillation and tachycardia (one patient) and hypotension (one patient). In the T80/A5 group, one patient discontinued because of ventricular extrasystoles. For all three patients, the adverse events were mild in intensity.

Adherence. The overall compliance during the double-blind period was $>80 \%$ in both treatment groups; $2(1.3 \%)$ of the 149 patients in the T80/A5/H12.5 group and none of the 160 patients in the T80/A5 group had a compliance rate of $<80 \%$.

Vital signs and other safety parameters. Following the 8-week treatment period, no clinically relevant changes in DBP, SBP or pulse rate following a position change were detected in either group compared with reference baseline. Furthermore, the seated pulse rate and pulse rate after a position change remained relatively unchanged at week 8 (Supplementary Table 4). Overall, both T80/A5/H12.5 and T80/A5 were well tolerated. 


\section{DISCUSSION}

Hypertension is a disease with a high incidence in Japan. The currently used monotherapies, including ARBs, CCBs and diuretics, are effective only in a limited proportion of patients. For those who remain resistant to therapy, a combination of antihypertensive agents is required. ${ }^{1}$ The aim of this study was to investigate the efficacy and safety of a combination of T80/A5/H12.5 for the treatment of hypertension in patients with an inadequate response to telmisartan $80 \mathrm{mg}$ and amlodipine $5 \mathrm{mg}$. The results indicate that the T80/A5/H12.5 group achieved significantly greater reductions in seated DBP and SBP relative to those achieved in the T80/A5 group. In addition, significantly more patients achieved DBP and SBP control in the T80/A5/H12.5 group compared with patients treated with T80/A5.

The adverse events reported in this study were mild or moderate in severity, and those events were already observed in those receiving monotherapy or two-agent combination therapy. The incidence of drug-related adverse events was higher in the T80/A5/H12.5 group than in the T80/A5 group. The frequent drug-related adverse events in the T80/A5/H12.5 group were elevated uric acid levels and hyperuricemia. The cause of the increase in uric acid can likely be explained by the addition of hydrochlorothiazide, because elevated uric acid is a common side effect of diuretics. ${ }^{4}$ These adverse events were manageable and did not pose a health risk.

In this study, each patient received two tablets: T80/A5 fixed-dose combination and H12.5 or T80/A5 fixed-dose combination and placebo. It is possible that a single tablet T80/A5/H12.5 combination treatment would improve patient adherence. Previous studies reported that the fixed combination of valsartan/amlodipine/ hydrochlorothiazide or olmesartan/amlodipine/hydrochlorothiazide improved adherence and persistence compared with administration of each drug as individual tablets. ${ }^{5-7}$ This suggests that the fixed combination of T80/A5/H12.5 may increase the adherence relative to a combination of three individual treatments.

A similarly designed study of a combination of losartan $50 \mathrm{mg}$, hydrochlorothiazide $12.5 \mathrm{mg}$ and amlodipine $5 \mathrm{mg}$ compared with coadministration of losartan $50 \mathrm{mg}$ plus amlodipine $5 \mathrm{mg}$ in Japanese patients with essential hypertension did not demonstrate a significant difference in DBP reduction with hydrochlorothiazide $12.5 \mathrm{mg}$ at week 8 (additional effect of $-1.1 \mathrm{~mm} \mathrm{Hg}$; 95\% CI: -2.7 to $0.6 ; P=0.205$ ). ${ }^{8}$ However, there was a significant additional effect of hydrochlorothiazide $12.5 \mathrm{mg}$ on SBP reduction at week $8(-3.2 \mathrm{~mm} \mathrm{Hg} ;-5.7$ to -0.8 ; $P=0.011){ }^{8}$ The difference between the results of the losartan study and ours is possibly because of the profile of ARBs, that is, usual dosage range, terminal half-life and volume of distribution. ${ }^{9}$ The high-dose ARB with a large volume of distribution is suitable to completely inhibit the renin-angiotensin system activated by CCBs and diuretics. ${ }^{10}$ Among ARBs, telmisartan is a long-acting antihypertensive drug that may provide sustained reductions in blood pressure throughout a $24-\mathrm{h}$ dosing period. ${ }^{9,11}$

The results of this study should be considered in light of its limitations. The main limitation of this study was that the mean age of the patients was 54.7 years and only $14.6 \%$ of patients were $\geqslant 65$ years old. Therefore, the efficacy and safety profiles of T80/A5/H12.5 in older patients could not be determined. The study was also only conducted in institutions within Japan, and hence the results are not generalizable to populations of other ethnicities. Another limitation was the short duration of the study, as 8 weeks may not have been sufficient to observe the full benefit provided by the combination therapy, or the benefits may have been reduced over a longer period. Finally, we did not include patients with severe impaired kidney function (estimated glomerular filtration rate $<30 \mathrm{ml} \mathrm{min}^{-1} 1.73 \mathrm{~m}^{-2}$ ) who may also require combination therapies to manage hypertension.

\section{CONCLUSIONS}

The results from this phase III study indicate that the combination of telmisartan, amlodipine and hydrochlorothiazide is more effective than that of only telmisartan and amlodipine at reducing blood pressure in hypertensive patients. The addition of hydrochlorothiazide to a ARB/CCB combination therapy provides clinicians with a suitable treatment option for patients unresponsive to ARB/CCB combinations. This triple combination therapy is effective and well tolerated.

\section{CONFLICT OF INTEREST}

$\mathrm{JH}$ received consulting and lecture fees and/or research funding from Astellas Pharmaceutical, Nippon Boehringer Ingelheim, Mochida Pharmaceutical, Daiichi Sankyo, Sumitomo Dainippon Pharmaceutical, MSD, Novartis Pharmaceutical, Teijin Pharmaceutical, and Takeda Pharmaceutical. IK has received lecture fees, research funding and/or scholarship grants from Daiichi Sankyo, Sumitomo Dainippon Pharmaceutical, Takeda Pharmaceutical, Mitsubishi Tanabe Pharmaceutical, Nippon Boehringer Ingelheim, Ono Pharmaceutical, Astellas Pharmaceutical, Teijin Pharmaaceutical, Sanofi, Kowa Company, Bayel Yakuhin and TOA EIYO. TO declares no conflict of interest. JH and IK were the Medical Experts and TO was the Coordinating Investigator of this study. KS, GL, AT, HI, DK and SN are employees of Nippon Boehringer Ingelheim.

\section{ACKNOWLEDGEMENTS}

We thank all investigators involved in this study: Shinichi Higashiue, MD (Kishiwada Tokushukai Hospital, Cardiovascular Surgery, Osaka, Japan); Kousuke Mabuchi, MD (Kanda Clinic, Internal Medicine, Tokyo, Japan); Shigeo Sawai, MD (Sawai Medical Clinic, Internal Medicine, Tokyo, Japan); Fumiki Oh, MD (Shinden Higashi Clinic, Internal Medicine, Miyagi, Japan); Isao Uchimura, MD (Chiyodahoujin Clinic, Internal Medicine, Tokyo, Japan); Masafumi Sugawara, MD (Dynamedical Nezu Clinic, Internal Medicine, Tokyo, Japan); Yoshihiro Tokeshi, MD (Chubu Tokushukai Hospital, Internal Medicine, Okinawa, Japan); Satoshi Inoue, MD (OCROM Clinic, Osaka, Japan); Katsuji Hashimoto, MD (AMC Nishiumeda Clinic, Internal Medicine, Osaka, Japan); Yoji Takatsuka, MD (Motomachi Takatsuka Naika Clinic, Internal Medicine, Kanagawa, Japan); Hiroshi Shimomura, MD (Musashino Polyclinic, Internal Medicine, Tokyo, Japan); Kazuko Takahashi, MD (Takahashi Clinic, Internal Medicine, Hokkaido, Japan); Fumihiko Takeda, MD (Riverside clinic, Internal Medicine, Hokkaido, Japan); Sohji Nagase, MD (Nagase Medical Clinic, Internal Medicine, Ibaraki, Japan); Toru Kihara, MD (Fukuoka Rehabilitation Hospital, Internal Medicine, Fukuoka, Japan); Toshiki Fukui, MD (NTT West Takamatsu Hospital, Internal Medicine, Kagawa, Japan); Takafumi Kondo, MD (Kaisei Hospital, Internal Medicine, Kagawa, Japan); Arihiro Kiyosue, MD (Tokyo-Eki Center-building Clinic, Internal Medicine, Tokyo, Japan); Takahiko Tokuyama, MD (Tokuyama Clinic, Internal Medicine, Chiba, Japan); Akira Numata, MD (Ikebukuro Metropolitan Clinic, Internal Medicine, Tokyo, Japan); Atsushi Hirayama, MD (Nihon University Itabashi Hospital, Division of Cardiovascular Medicine, Tokyo, Japan); Hideki Takizawa, MD (Teine Keijinkai Clinic, Kidney Internal Medicine, Hokkaido, Japan); Teruaki Mita, MD (Mita Internal Medicine Cardiology Clinic, Internal Medicine, Hokkaido, Japan); Chiaki Noguchi, MD (Shinkoiwa Ekimae Clinic, Internal Medicine, Tokyo, Japan); Yuichiro Nakamura, MD (Nakamura Cardiovascular Clinic, Cardiovascular Medicine, Fukuoka, Japan); Norimasa Sato, MD (Umeda Oak Clinic, Internal Medicine, Osaka, Japan); Takayuki Azuma, MD (Yaesu Sakura-dori Clinic, Internal Medicine, Tokyo, Japan); Nobuaki Onizawa, MD (Kaijo Bill Clinic, Internal Medicine, Tokyo, Japan); Koichi Yamamoto, MD (Osaka University Hospital, Presbyo-hypertension, Osaka, Japan); Yoshinori Seko, MD (The Institute for Adult Diseases, Asahi Life Foundation, Cardiovascular Internal Medicine, Tokyo, Japan). Treatments were developed by Nippon Boehringer Ingelheim. This study was funded by Nippon Boehringer Ingelheim. We thank Mami Mori of Nippon Boehringer Ingelheim for publication management and editorial support, and John Gibbins of Edanz 
Group for providing medical writing support. This medical writing assistance was funded by Nippon Boehringer Ingelheim.

1 Shimamoto K, Ando K, Fujita T, Hasebe N, Higaki J, Horiuchi M, Imai Y, Imaizumi T, Ishimitsu $\mathrm{T}$, Ito $\mathrm{M}$, Ito $\mathrm{S}$, Itoh $\mathrm{H}$, Iwao $\mathrm{H}$, Kai H, Kario $\mathrm{K}$, Kashihara N, Kawano $\mathrm{Y}$, Kim-Mitsuyama S, Kimura G, Kohara K, Komuro I, Kumagai H, Matsuura H, Miura K, Morishita R, Naruse M, Node K, Ohya Y, Rakugi H, Saito I, Saitoh S, Shimada K, Shimosawa T, Suzuki H, Tamura K, Tanahashi N, Tsuchihashi T, Uchiyama M, Ueda S, Umemura S, Japanese Society of Hypertension Committee for Guidelines for the Management of Hypertension. The Japanese Society of Hypertension Guidelines for the Management of Hypertension (JSH 2014). Hypertens Res 2014; 37: 253-390.

2 Teramoto T, Toshiro F. Japan guideline assessment panel-2 (J-GAP2). Prog Med (Tokyo) 2010; 30: 1437-1449.

3 Nakagawa M, Masuda A, Ura N, Tanaka S. Transition of achievement rate for lowering blood pressure target level and situation of prescription for antihypertensive drug. J Blood Press 2011; 18: 73-77.

4 Moore MJ, Gong Y, Hou W, Hall K, Schmidt SOF, Curry RW, Beitelshees AL, Chapman A, Turner ST, Schwartz GL, Bailey K, Boerwinkle E, Gums JG, Cooper-DeHoff RM, Johnson JA. Predictors for glucose change in hypertensive participants following short-term treatment with atenolol or hydrochlorothiazide. Pharmacotherapy 2014; 34: 1132-1140.

5 Oparil S, Melino M, Lee J, Fernandez V, Heyrman R. Triple therapy with olmesartan medoxomil, amlodipine besylate, and hydrochlorothiazide in adult patients with hypertension: the TRINITY multicenter, randomized, double-blind, 12-week, parallel-group study. Clin Ther 2010; 32: 1252-1269.

6 Mohan JC, Jain R, Chamle V, Bhargava A. Short term safety and tolerability of a fixed dose combination of olmesartan, amlodipine and hydrochlorothiazide. J Clin Diagn Res 2015; 9: OC10-OC13.

7 Machnicki G, Ong SH, Chen W, Wei ZJ, Kahler KH. Comparison of amlodipine/ valsartan/hydrochlorothiazide single pill combination and free combination: adherence, persistence, health care utilization and costs. Curr Med Res Opin 2015; 31: 2287-2296.

8 Rakugi H, Tsuchihashi T, Shimada K, Numaguchi $\mathrm{H}$, Nishida C, Yamaguchi $\mathrm{H}$, Shirakawa M, Azuma K, Fujita KP. Add-on effect of hydrochlorothiazide $12.5 \mathrm{mg}$ in Japanese subjects with essential hypertension uncontrolled with losartan $50 \mathrm{mg}$ and amlodipine 5 mg. Hypertens Res 2015; 38: 329-335.

9 Chrysant SG, Chrysant GS, Desai A. Current status of angiotensin receptor blockers for the treatment of cardiovascular diseases: focus on telmisartan. J Hum Hypertens 2005; 18: $172-183$.

10 Konoshita TM, Makino Y, Kimura T, Fuji M, Wakahara S, Arakawa K, Inoki I, Nakamura H, Miyamori I, Genomic Disease Outcome Consortium (G-DOC) Study Investigators. A new-generation N/L-type calcium channel blocker leads to activation of the renin-angiotensin system compared with conventional $L$ type calcium channel blocker. J Hypertens 2010; 28: 2156-2160.

11 Neutel J, Smith DH. Evaluation of angiotensin II receptor blockers for 24-hour blood pressure control: meta-analysis of a clinical database. J Clin Hypertens 2003; 5: $58-63$.

(c) (1) (2) $\odot$ This work is licensed under a Creative Commons Attribution-NonCommercial-NoDerivs $\quad 4.0$ International License. The images or other third party material in this article are included in the article's Creative Commons license, unless indicated otherwise in the credit line; if the material is not included under the Creative Commons license, users will need to obtain permission from the license holder to reproduce the material. To view a copy of this license, visit http://creativecommons.org/licenses/ by-nc-nd/4.0/

(C) The Author(s) 2017

Supplementary Information accompanies the paper on Hypertension Research website (http://www.nature.com/hr) 